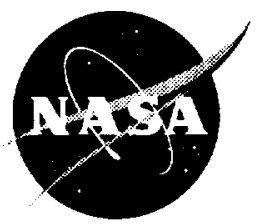

\title{
A High Frequency Model of Cascade Noise
}

Edmane Envia

Lewis Research Center, Cleveland, Ohio

Prepared for the

Fourth Aeroacoustics Conference

cosponsored by the American Institute of Aeronautics and Astronautics and the Confederation of European Aerospace Societies

Toulouse, France, June 2-4, 1998

National Aeronautics and

Space Administration

Lewis Research Center 
Available from

NASA Center for Aerospace Information 7121 Standard Drive Hanover, MD 21076

Price Code: A03

National Technical Information Service 5285 Port Royal Road Springfield, VA 22100

Price Code: A03 


\title{
A HIGH FREQUENCY MODEL OF CASCADE NOISE
}

\author{
Edmane Envia* \\ Acoustics Branch. NASA Lewis Research Center \\ Cleveland, Ohio, USA
}

\begin{abstract}
Closed form asymptotic expressions for computing high frequency noise generated by an annular cascade in an infinite duct containing a uniform flow are presented. There are two new elements in this work. First, the annular duct mode representation does not rely on the often-used Bessel function expansion resulting in simpler expressions for both the radial eigenvalues and eigenfunctions of the duct. In particular, the new representation provides an explicit approximate formula for the radial eigenvalues obviating the need for solutions of the transcendental annular duct eigenvalue equation. Also, the radial eigenfunctions are represented in terms of exponentials eliminating the numerical problems associated with generating the Bessel functions on a computer. The second new element is the construction of an unsteady response model for an annular cascade. The new construction satisfies the boundary conditions on both the cascade and duct walls simultaneously adding a new level of realism to the noise calculations. Preliminary results which demonstratc the effectiveness of the new elements are presented. A discussion of the utility of the asymptotic formulas for calculating cascade discrete tone as well as broadband noise is also included.
\end{abstract}

\section{INTRODUCTION}

Prediction of fan noise continues to be an integral part of the efforts aimed at analysis and suppression of turbofan engine noise. To that end, a number of schemes have been developed to calculate the contribution of various fan-associated noise sources. But, whether the goal is to predict rotor inlet noise (e.g., Mani [1973|), cascade self-noise (e.g.. Glegg [1996]) or rotor-stator interaction noise (e.g. Envia et. al, [1996]), all of these schemes are predicated on computing the acoustic response of a blade row to incident flow perturbations

In strictly non-CFD based methods, which continue to be the principal tools of fan noise prediction, the blade row acoustic response is computed using simplified versions of the cascade geometry and/or the governing equations. At the simplest end of the spectrum, approximations in both the geometry and governing equations yield analytical or semi-analytical expressions for the acoustic response. Generally speaking, the approximations involve replacing the cascade with zero thickness flat plates and linearizing the governing equations about a uniform base flow. In this class of methods the acoustic response can be determined either directly by solving the governing equations or indirectly through the use of the annular duct Green's functions which couple the unsteady surface pressure distribution on the cascade to the duct acoustic modes.

However, even at that level of approximation. exact solutions are still difficult to obtain, so further simplifications are made. Some simplifications are based on replacing the annular cascade by a rectilinear one at some representative radius and computing the acoustic response for the resulting system (e.g.. Goldstein (1976]). In other simplifications, the annular geometry is retained but the cascade unsteady pressure distribution (henceforth, called the cascade unsteady response) is computed at a number of spanwise 2D strips and the resulting distribution assembled and coupled to the duct acoustic modes (e.g., Ventres et. al [1982]).

While in both cases useful information regarding general trends can be obtained, it is highly desirable to retain as much of the cascade and unsteady response three-dimensionality as possible. Such 3D models have been explored by, among others. Namba [1977], Kobayashi [1978] and Schulten [1993], but

\footnotetext{
*Aerospace Engineer, Senior Member. AIAA
} 
these approaches generally require the use of collocation techniques or numerical solutions of partial differential equations.

In this paper, a closed form approximate model of cascade noise is presented which takes into account the annular geometry of the blade row throughout the analysis. The noise field is computed by coupling the cascade unsteady response to the duct acoustic modes in the standard manner. However, asyınptotic methods have been employed to circumvent the need for numerical solution of the governing equations by tailoring the model to the high frequency noise regime. Given the current interest in high-speed fans, this high frequency specialization, far from being a limitation, is in fact an asset since the numerically based methods have inherent difficulties handling the high frequency response regime

In what follows, the development of two new elements in modeling of annular cascade noise will be presented. The first element involves a representation of annular duct modes that is not predicated on the use of the Bessel functions. The second new element addresses the construction of an unsteady response to incident flow perturbations (i.e., gusts) for an annular cascade where the finite-span effects as well as the gust three-dimensionality are taken into account. In each case, intermediate results will be presented which show the effectiveness of the new approximations. The two elements are then combined to provide the desired high frequency model of cascade noise. The paper is concluded with a discussion of the utility of the new model for predicting cascade tone and broadband noise.

\section{ANALYSIS}

The cascade is modeled as an ensemble of zero camber and thickness flat plates enclosed in an infinite hard-wall annular duct within which exits a uniform and isentropic medium. As shown in Figure 1. two coordinate systems will be employed in this work. The global system $(x, r, \theta)$ is cylindrical polar and is aligned with the axis of the duct with $\theta$ measured positive counterclockwise. The local system $(\xi, \eta, \zeta)$ is Cartesian and is aligned with the reference (i.e., zeroth) airfoil. With no loss of generality, we assume that the $\theta=0$ line and $\zeta$ axes are coincident and along the leading edge of the reference airfoil (see Figure 1). The relationship between the two systems is given by
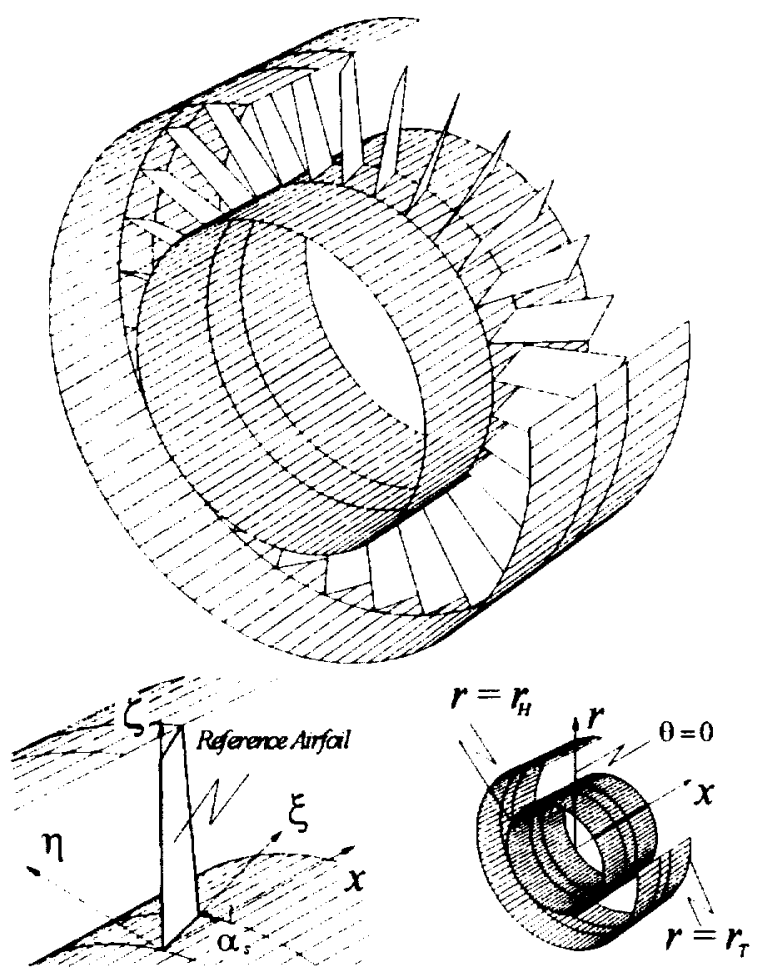

Figure I. Cascade geometry and coordinates systems.

$$
\begin{aligned}
& \xi=x \cos \alpha_{s}+r \theta \sin \alpha_{s} \\
& \eta=-x \sin \alpha_{s}+r \theta \cos \alpha_{s} \\
& \zeta=\frac{r-\sigma}{1-\sigma}
\end{aligned}
$$

where $\alpha_{s}$ is the stagger angle of the cascade. Eq. (1) holds as long as $\theta$ is small (i.e., near the reference airfoil). The axial and radial coordinates are nomalized by the tip radius, as are the local coordinates. $\sigma=r_{H} / r_{T}$ is the hub-to-tip radius ratio with $r_{H}$ and $r_{T}$ denoting the hub and tip radii. In what follows, it is most convenient to describe the duct acousuc field in terms of the global coordinate system, while the building block of the cascade unsteady response is most easily derived in the local coordinate system.

The representation of the duct acoustic field is outlined first followed by the construction of the cascade unsteady response model. In both instances, we will take advantage of asymptotic methods to simplify the analysis. The connection between the two elemer ts is then established using the Green's function method 


\section{Annular Duct Mode Expansion}

For the duct mode acoustic field calculations, we assume the base flow to be axial. The propagation of acoustic pressure waves in such a system is governed by the convected wave equation which, in the duct coordinates, takes the form

$$
\begin{aligned}
& \left(\nabla^{2}-a_{0}^{-2} \frac{D_{0}^{2}}{D t^{2}}\right) p^{\prime}=0 \\
& \nabla^{2}=\frac{\partial^{2}}{\partial x^{2}}+\frac{1}{r} \frac{\partial}{\partial r}\left(r \frac{\partial}{\partial r}\right)+\frac{1}{r^{2}} \frac{\partial^{2}}{\partial \theta^{2}} \\
& \frac{D_{0}}{D t}=\frac{\partial}{\partial t}+U \frac{\partial}{\partial x} \\
& \left.\frac{\partial p^{\prime}}{\partial r}\right|_{\text {Duct }}=0
\end{aligned}
$$

$p^{\prime}(x, r, \theta . t)$ is the acoustic pressure, $a_{0}$ the nominal speed of sound of the medium and $U$ the base flow.

Using the normal mode expansion approach, the most general form of $\boldsymbol{p}^{\prime}$ with harmonic time dependence is given by

$$
p^{\prime}(x, r, \theta, t)=\sum_{m=-\infty}^{\infty} \bar{p}_{m}^{\prime}(x, r) e^{i(m \theta-\omega t)}
$$

where $\omega$ is the frequency and $\boldsymbol{m}$ the spinning (i.e., circumferential) mode order. Depending on whether one is concemed with tone noise or broadband noise, there will be a sum or an integral over all frequencies of interest. The form of pressure given by Eq. (3), simplifies Eq. (2a) to

$$
\begin{aligned}
& {\left[\beta^{2} \frac{\partial^{2}}{\partial x^{2}}+\frac{1}{r} \frac{\partial}{\partial r}\left(r \frac{\partial}{\partial r}\right)-\frac{m^{2}}{r^{2}}+2 i k M \frac{\partial}{\partial x}+k^{2}\right] \bar{p}_{m}^{\prime}=0} \\
& k=\frac{\omega r_{T}}{a_{0}}, \quad M=\frac{U}{a_{0}}, \quad \beta=\sqrt{1-M^{2}}
\end{aligned}
$$

Here $\boldsymbol{k}$ is the reduced frequency and $\boldsymbol{M}$ is the Mach number of the mean flow. Introducing

$$
\bar{p}_{m}^{\prime}(x, r)=f_{m}(x) g_{m}(r)
$$

leads to separate equations in $x$ and $r$ given by

$$
\begin{aligned}
& {\left[\beta^{2} \frac{d^{2}}{d x^{2}}+2 i k M \frac{d}{d x}+\left(k^{2}-\kappa^{2}\right)\right] f_{m}=0} \\
& {\left[\frac{d^{2}}{d r^{2}}+\frac{1}{r} \frac{d}{d r}+\kappa^{2}-\frac{m^{2}}{r^{2}}\right] g_{m}=0} \\
& \left.\frac{d g_{m}}{d r}\right|_{\text {duct }}=0
\end{aligned}
$$

The Ierm $\kappa^{2}$ in these equations is the separation constant. We solve for the second equation first and postpone the solution of the first one until later in the analysis.

Since Eq. (7a) is the Bessel's Equation, it is usually solved in terms of the Bessel Functions of the First and Second kind of index $m$. The corresponding boundary value problem (i.e., Eq. (7b)) then leads to a transcendental equation for the eigenvalues of the annular duct (i.e., the permissible values of $x$ ). The eigenvalues are generally obtained numerically by solving the transcendental equation iteratively. But here we pursue a different approach for computing $\boldsymbol{\kappa}$ 's.

The integrating factor $e^{-\frac{1}{2} \int \frac{d r}{r}}=r^{-1 / 2}$ for Eq. (7a) suggests the following change of dependent variable $g_{m}(r)=r^{-1 / 2} \widetilde{g}_{m}(r)$. Rewriting Eqs. (7a \& b) in terms of the new dependent variable yields

$$
\begin{aligned}
& {\left[\frac{d^{2}}{d r^{2}}+w^{2}(r)\right] \widetilde{\boldsymbol{g}}_{m}=0} \\
& w(r)=\sqrt{\kappa^{2}-\frac{m^{2}}{r^{2}}+\frac{1}{4 r^{2}}} \\
& \left.\left(\frac{d}{d r}-\frac{1}{2 r}\right) \widetilde{\boldsymbol{g}}_{m}\right|_{r=\sigma, 1}=0
\end{aligned}
$$

The distribution of the eigenvalues of the Bessel's Equation is such that the smallest eigenvalue in the sequence is always greater than the index (i.e.. $\operatorname{Min}(\kappa) \geq \boldsymbol{m})$ with the other elements of the sequence growing rapidly larger than $m$. Therefore, as long as $\sigma>0$ (i.e., $\left.r_{H} \neq 0\right), w(r)$ will vary slowly with $r$ for given $\kappa$ and $m$. This suggests that the WKB method (Olver [1974]) may provide a reasonable 
approximation to the solution of Eq. (8a) and its eigenvalues. The accuracy of the approximation will. of course, depend on the behavior of $w(r)$. but as it will be shown the proposed approximation is surprisingly accurate. especially, for higher order eigenvalues as long as $\sigma$ is not too close to zero.

The asymptotic formula for the eigenvalues is derived in Appendix 1. The final resull is

$$
\kappa_{m n} \doteq \frac{n \pi \sigma+\sqrt{(n \pi \sigma)^{2}+\sigma(1-\sigma)^{2}\left(2 m^{2}+3 / 2\right)}}{2 \sigma(1-\sigma)}
$$

where $\boldsymbol{\kappa}_{m n}$ is the non-dimensional eigenvalue and $n=0,1,2, \cdots$ its "radial" index set corresponding to each circumferential index $m$. The symbol $\doteq$ indicates that $\boldsymbol{x}_{m n}$ is an approximation to the exact cigenvalue.

Eq. (9) is a simple formula but a remarkably accurate one as is shown in Figure 2. Here the exact (open bars) and approximate (solid bars) radial eigenvalues are plotted for two circumferential mode orders $m=6$ and $m=30$ for a hub-to-tip radius ratio of 0.5 . The first 20 eigenvalues are shown in each case. Maximum approximation error is around 15\% for low values of the radial index but as $n$ increases the error diminishes rapidly. Given that the exact eigenvalues must be determined numerically for each combination of $\boldsymbol{m}, \boldsymbol{n}$ and $\sigma$, the advantage of having an explicit formula for computing the duct eigenvalues accurately becomes apparent.

The corresponding eigenfunctions, also derived in Appendix 1, are given by the expression

$$
\begin{aligned}
& \Phi_{m n}(r) \doteq \sqrt{\frac{1}{\kappa_{m n} r}}\left(1-\frac{1-4 m^{2}}{16\left(\kappa_{m n} r\right)^{2}}\right) \\
& \times \Re\left(e^{i \psi_{0}\left(\kappa_{m n} \cdot r\right)}+\frac{\sigma\left(\kappa_{m n}, \sigma\right)}{\sigma^{*}\left(\kappa_{m n}, \sigma\right)} e^{2 i \psi_{0}\left(\kappa_{m . n}, \sigma\right)} e^{-i \psi_{0}\left(\kappa_{m, r}, r\right)}\right)
\end{aligned}
$$

$\psi_{a}(\kappa, r)=\kappa r+\frac{4 m^{2}-1}{8 \kappa r}$

$$
\varpi(\kappa, \sigma)=2 \sigma \psi_{a}^{\prime}(\kappa, \sigma)+i
$$

\footnotetext{
- The exact eigenvalues are solutions of the transcendental equation $J_{m}^{\prime}\left(\kappa_{m n} \sigma\right) Y_{m}^{\prime}\left(\kappa_{m n}\right)-J_{m}^{\prime}\left(\kappa_{m n}\right) Y_{m}^{\prime}\left(\kappa_{m n} \sigma\right)=0$.
}
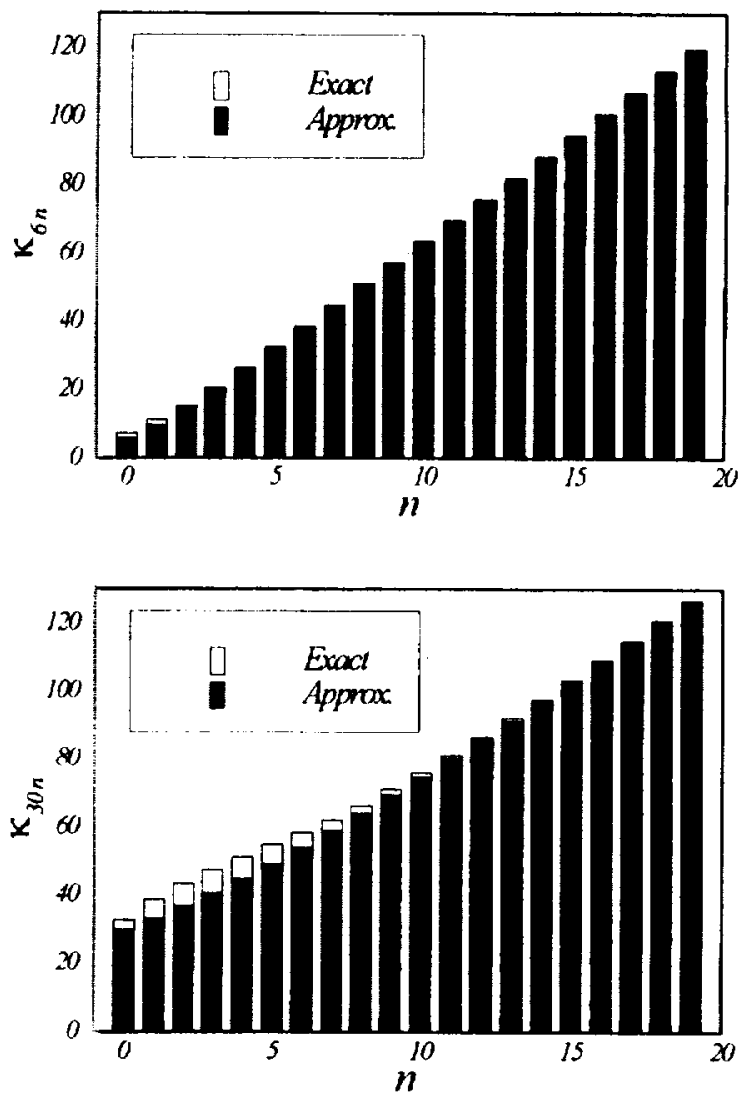

Figure 2. Comparisons of exact and approximate radial eigenvalue distributions for two circumferential mode orders. For this calculation $\sigma=0.5$.

The prime in Eq. (10c) denotes differentiation with respect to the radial coordinate $r$ and the symbol $R$ indicalt:s the real pan.

Eq. (10a) provides an efficient altemative to the exact" annular duct eigenfunctions which generally require recursive computation of the Bessel functions of various order. In Figure 3, a comparison of the exact and approximate eigenfunctions is shown. The first four normalized radial eigenfunctions are plotted for the circumferential mode $m=6$ at $\sigma=0.5$. Except for the lowest order approximate eigenfunction. which shows marked difference from the exact one, the rest are consistently close to the exact eigenfunctions with the agreement improving as $n$ increases. The fourth one, in particular, is virtually indistinguishable from the exact eigenfunction. These results, and those shown $n$ Figure 2, demonstrate that the formulas given by Eqs. (9) and (10a) provide useful approximation that

\footnotetext{
"- The exact radial eigenfunctions are given by the expression $\Phi_{m n}(r)=J_{m}\left(\kappa_{m n} r\right)-\frac{J_{m}^{\prime}\left(\kappa_{m n} \sigma\right)}{Y_{m}^{\prime}\left(\kappa_{m n} \sigma\right)} Y_{m}\left(\kappa_{m n} r\right)$
} 

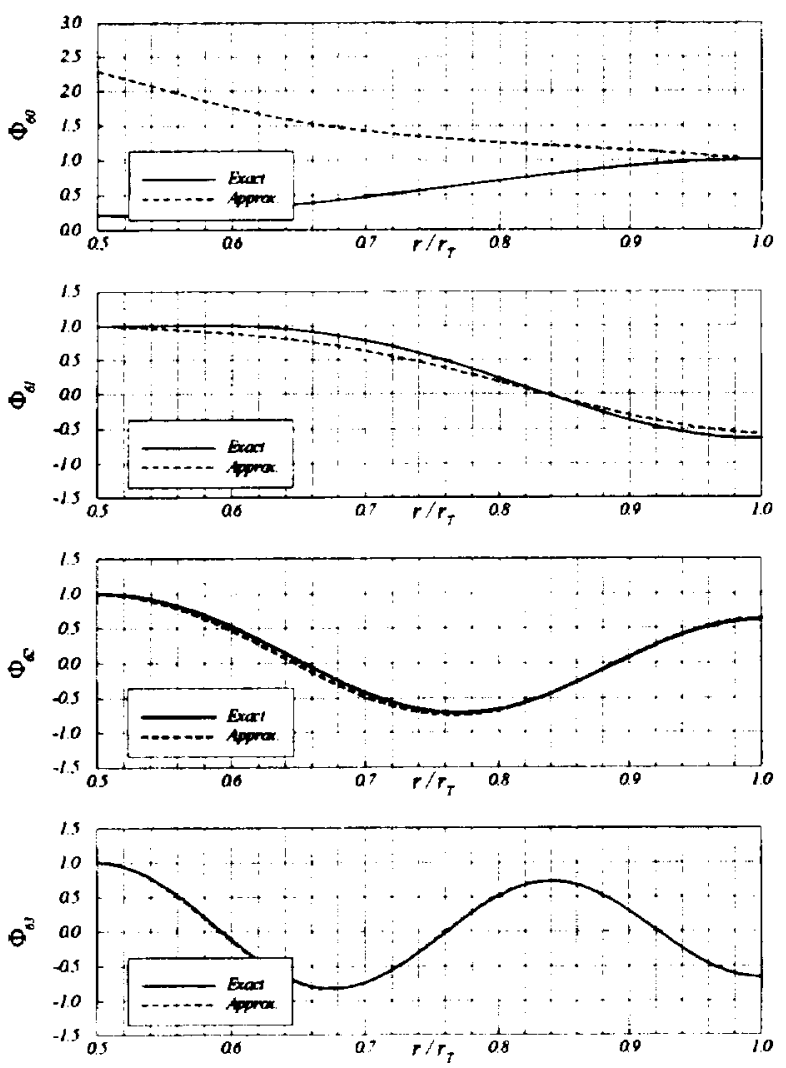

Figure 3. Comparisons of exact and approximate radial eigenfunctions (normalized) for the circumferential mode order $m=6$ with $\sigma=0.5$. Eigenfunctions corresponding to the first four radial orders are shown.

can be used in place of the exact eigenvalues and eigenfunctions.

Eqs. (9) and (10a) together constitute the first of two main results of this paper. A detailed discussion of the behavior and analytic structure of these approximate eigenvalues and eigenfunctions is postponed to a future time.

With the solution of the radial eigenvalue problem determined, the most general solution of Eq. (7a) is given by

$$
g_{m}(r)=\sum_{n=0}^{\infty} C_{m n} \Phi_{m n}(r)
$$

where $C_{m m}$ 's are acoustic mode amplitudes that will be determined later.

Now, the solutions of Eq. (6) can be readily written down as

$$
\begin{gathered}
f_{m}(x)=e^{i k_{m}^{ \pm} x} \\
k_{m n}^{ \pm}=-k \frac{M}{\beta^{2}} \pm \frac{1}{\beta} \sqrt{\frac{k^{2}}{\beta^{2}}-\kappa_{m n}^{2}}
\end{gathered}
$$

where the plus sign indicates downstream running acoustic waves and the minus sign upstream nunning ones. Combining the expressions for $f_{m}$ and $g_{m}$, the general representation for the acoustic pressure field inside the duct is given by

$$
p^{\prime}(x, r, \theta, t)=\sum_{m=\infty}^{\infty} \sum_{n=1)}^{\infty} C_{m n} \Phi_{m n}(r) e^{i\left(k_{m n}^{ \pm} x+m \theta-\omega t\right)}
$$

For a given geometry and operating condition, only a finite number of terms in the double infinite sums will constitute propagating waves. Waves (i.e., modes), for which $\boldsymbol{k}_{m n}^{ \pm}$is complex, are cut-off and do not contribute to the acoustic field.

\section{Annular Cascade Unsteady Response Model}

The complications introduced by the annular geometry of the cascade generally require numerical solution of the equations governing the unsteady pressure distribution on the cascade. To avoid a numerical treatment, the unsteady response will be constructed in a manner similar to that developed by Envia and Kerschen [1986] for the radiated noise from a rectilinear cascade.

Taking advantage of the weak coupling between the adjacent airfoils at high frequencies. these authors envisaged the cascade noise field as a sum over the individual airfoil fields with the blade-to-blade periodicity enforced. Here we modify the procedure to account for the annular geometry and deduce the unsteady pressure distribution on the cascade from the local acoustic field. The solution is of course approximate, but as was shown in the above reference, the approximation provides quite reasonable accuracy at high frequencies.

The starting point for construction of the cascade response is the single airfoil solution. This solution is derived for the reference (i.e., zeroth) airfoil using the Wiener-Hopf technique in terms of "leading edge" and "trailing edge" responses that are combined to produce the response of the airfoil to incident flow perturbations. The details of the derivation, as well as a discussion of some sample calculations, are given in 
Appendix 2. Here we present the final results expressed in the duct coordinate system. The solution is given by

$$
\begin{aligned}
& p_{0}^{\prime}(x, r, \theta, t)=\sum_{\mu=-\infty}^{\infty} \sum_{v=0}^{\infty} \sum_{t=0}^{\infty} A_{\mu v} \cos \left(l \pi \frac{r-\sigma}{1-\sigma}\right) e^{-i \omega t} \\
& \times\left[G_{1}(x, r, \theta) e^{i t_{1}(x, r, \theta)}+G_{2}(x, r, \theta) e^{i \tau_{2}(x, r, \theta)}\right] \quad(14 \mathrm{a}) \\
& \tau_{j}(x, r, \theta)=\chi R_{j}(x, r, \theta) \\
& -\gamma_{\xi} \frac{M_{\xi}^{2}}{\beta_{\xi}}\left(x_{j} \cos \alpha_{s}+r \theta \sin \alpha_{s}\right) \quad(14 \mathrm{~b}) \\
& R_{j}=\sqrt{x_{j}^{2}+(r \theta)^{2}}, \quad x_{j}=x-(j-1) c
\end{aligned}
$$

where $c$ is the airfoil chord. The definitions for all the variables appearing in these equations can be found in Appendix 2. The triple summation provides the response over all possible gust modes that might exist inside the annulus as discussed in the beginning of Appendix 2. It should be emphasized that, at this point in the analysis, $\boldsymbol{p}_{0}^{\prime}$ is the unsteady pressure field in the vicinity of the zeroth airfoil and not just on its surface alone. After the cascade solution is constructed the final expressions will be evaluated on the surface of the cascade.

Since the circumferential positions of any airfoil in the cascade is related to the circumferential position of the reference airfoil through

$$
\theta_{q}=\theta-q \varphi, \quad q=0, \pm 1, \pm 2, \cdots
$$

where $\varphi=2 \pi / N$ ( $N$ is the number of airfoils in the cascade). the acoustic response of any airfoil in the cascade can be related to the response of the reference airfoil. The required relationship can be established by noting that. on a per mode basis, the form of the incident gust on the $q$ th airfoil expressed in the zeroth airfoil coordinates, is related to the form of the gust on the zeroth airfoil expressed in the $q$ th airfoil coordinate system through

$$
\left.u_{g_{\mu v}}(x, r, \theta, t)\right|_{q^{\text {th a arfoil }}}=\left.u_{g_{\mu v}}\left(x, r, \theta_{q}, t\right)\right|_{0^{\text {th airfoil }}} e^{i \mu q \varphi}
$$

where we have taken advantage of the circumferential dependence of the gust as given by Eq. (A2.1) in Appendix 2. This suggests that an identical connection must hold between the responses of other airfoils and the response of the reference airfoil. That is to say,

$$
p_{q}^{\prime}(x, r, \theta, t)=p_{0}^{\prime}\left(x, r, \theta_{q}, t\right) e^{i q \mu \varphi}
$$

Adding up the contribution of all airfoils we find

$$
p_{c}^{\prime}(x, r, \theta, t)=\sum_{q=-\infty}^{\infty} p_{0}^{\prime}\left(x, r, \theta_{q}, t\right) e^{i q \mu \varphi}
$$

where $\boldsymbol{p}_{c}^{\prime}$ is cascade response. The limits of the summation are set to infinity to ensure that the resulting solution and all of its derivatives are periodic around the annulus. Had we limited the sum to only $\boldsymbol{N}$ terms (corresponding to $N$ airfoils), only the periodicity of the solution itself would have been guaranteed. Furthermore, as it will shown later, the infinite sum in Eq. (18) ensures that all circumferential modes that can exist in the duct are accommodated.

Taking advantage of the properties of the Dirac delta function, Eq. (18) can be rewritten as

$$
p_{c}^{\prime}(x, \cdots, \theta, t)=\sum_{q=-\infty}^{\infty} \int_{-\infty}^{\infty} p_{0}^{\prime}(x, r, \Theta, t) e^{i q \mu \rho} \delta\left(\Theta-\theta_{q}\right) d \Theta
$$

Interchanging the order of summation and integration and using the Poisson Sum formula (see Carrier et. al [1966] the sum in Eq. (19) can be converted to an equiva ent sum (say, with index $\ell$ ) having the form

$$
\begin{aligned}
\rho_{c}^{\prime}(x, r, \theta, t) & =\frac{1}{\sqrt{2 \pi \varphi}} \sum_{\ell=-\infty}^{\infty} e^{i(\mu+2 \ell \pi / \varphi) \theta} \\
& \times \int_{-\infty}^{\infty} p_{0}^{\prime}(x, r, \Theta, t) e^{-i(\mu+2 \ell \pi / \varphi) \Theta} d \Theta
\end{aligned}
$$

Given the complicated form of $p_{0}^{\prime}(x, r, \Theta, t)$ (see $\mathrm{Eq}$. (14a)), the integral in Eq. (20) cannot be evalualed exactly, but it can be computed asymptotically using the method of steepest descent in the high frequency limit (i.e., for $k>1$ ). Substituting for $p_{0}^{\prime}(x, r, \Theta, t)$ and grouping terms together, Eq. (20) can be rewritten in canonical form as 


$$
\begin{aligned}
& p_{c}^{\prime}(x, r, \theta, t)=\frac{1}{\sqrt{2 \pi} \varphi} \sum_{\ell=-\infty}^{\infty} I_{\ell} e^{i(\mu+2 \ell \pi / \varphi) \theta} \\
& I_{\ell}=\int_{-\infty}^{\infty}\left[\Lambda_{1}(\Theta) e^{i \Psi_{1}(\Theta)}+\Lambda_{2}(\Theta) e^{i \Psi_{2}(\Theta)}\right] d \Theta
\end{aligned}
$$

where the phase functions $\Psi_{j}$ contain all the phase terms that depend in the variable $\Theta$ and the amplitude terms $\Lambda_{j}$ include everything else in Eq. (14a). The phase functions are given by

$$
\begin{aligned}
\Psi_{j}(\Theta)= & \chi \sqrt{x_{j}^{2}+(\mu \Theta)^{2}} \\
& -\gamma_{\xi} \frac{M_{\xi}^{2}}{\beta_{\xi}} \mu \sin \alpha_{s}-(\mu+2 \ell \pi / \varphi) \Theta
\end{aligned}
$$

with $x_{j}=x-(j-1) c$. The saddle points $\Theta_{j_{\mathrm{sp}}}$ (i.e., the roots of $\left.d \Psi_{j} / d \Theta=\Psi_{j}^{\prime}=0\right)$ are given by

$$
\Theta_{j_{\mathrm{sp}}}=\frac{\left[\gamma_{\xi} \frac{M_{\xi}^{2}}{\beta_{\xi}} \sin \alpha_{s}+\frac{1}{r}(\mu+2 \ell \pi / \varphi)\right] x_{j}}{r \sqrt{\chi^{2}-\left(\gamma_{\xi} \frac{M_{\xi}^{2}}{\beta_{\xi}} \sin \alpha_{s}+\frac{1}{r}(\mu+2 \ell \pi / \varphi)\right)^{2}}}
$$

Carrying out the remaining steps in the analysis leads to the asymptotic approximation of the integral $I$, The final result is given by

$$
\begin{aligned}
& I_{\ell} \cong \sqrt{2 \pi} e^{i \pi / 4} \\
& \times\left[\frac{\Lambda_{1}\left(\Theta_{1 \mathrm{sp}}\right)}{\sqrt{\Psi_{1}^{\prime \prime}\left(\Theta_{1 \mathrm{sp}}\right)}} e^{\prime \Psi_{1}\left(\Theta_{l_{p}}\right)}+\frac{\Lambda_{2}\left(\Theta_{2 \mathrm{sp}}\right)}{\sqrt{\Psi_{2}^{\prime \prime}\left(\Theta_{2 \mathrm{sp}}\right)}} e^{i \Psi_{2}\left(\Theta_{2 \mathrm{q}}\right)}\right] \\
& \Psi_{,}\left(\Theta_{J_{\mathrm{p}}}\right)=x_{j} \sqrt{\chi^{2}-\left(\gamma_{\xi} \frac{M_{\xi}^{2}}{\beta_{\xi}} \sin \alpha_{\mathrm{s}}+\frac{1}{r}(\mu+2 \ell \pi / \varphi)\right)^{2}}
\end{aligned}
$$

$$
\begin{aligned}
& \Psi_{j}^{\prime \prime}\left(\Theta_{s_{\mathrm{sp}}}\right) \\
& =\frac{r^{2}\left[\chi^{2}-\left(\gamma_{\xi} \frac{M_{\xi}^{2}}{\beta_{\xi}} \sin \alpha_{s}+\frac{1}{r}(\mu+2 \ell \pi / \varphi)\right)^{2}\right]^{3 / 2}}{\chi^{2} x_{j}}
\end{aligned}
$$

where $\Psi_{j}^{\prime \prime}$ is the second derivative of the phase function given in Eq. (22).

The waves implied by Eq. (24a) are cut-off whenever the square roots in the expressions for $\Psi_{,}\left(\Theta_{J_{\text {. }}}\right)$ (i.e., Eq. (24b)) become imaginary. In particular, consider the case for tone noise generated by the interaction of mean wakes of a $B$-bladed rotor with a $V$-vaned stator. For this situation the circumferential structure of the gust implies that $\mu=\wp B$ where $\wp$ is an integer. If the stagger angle $\alpha_{s}$ were zero, the cut-off condition for the lowest order spanwise mode (i.c., $l=0$ ) would imply

$$
\frac{\wp B \Omega_{r_{T}}}{a_{0} \beta}<\frac{1}{r}|\wp B+\ell V|
$$

where we have taken advantage of the following facts:

$$
\begin{aligned}
& \left.\chi\right|_{1=0}=k_{x} M / \beta=\omega r_{T} / a_{0} \beta, \\
& \omega=\wp B \Omega, \\
& \varphi=2 \pi / V
\end{aligned}
$$

and $\Omega$ is the fan rotational speed.

If the cut-off condition is satisfied for the lip radius, it will be satisfied for all other radii. Setting $r=1$ in Eq. (25) and re-arranging the resulting equation, we arrive at the familiar Tyler-Sofrin cut-off criterion (see Tyler and Sofrin [1962]);

$$
\left|\frac{\wp B+\ell V}{\wp B}\right|>\frac{M_{\mathrm{T}}}{\boldsymbol{B}}
$$

where $\boldsymbol{M}_{T}=\Omega \boldsymbol{r}_{T}$ is the tip Mach number of the rotor. Modes for which the above inequality holds are cut-off and do not contribute to the cascade response field. For higher order spanwise modes and/or non-zero stagger angles the cut-off condition is somewhat more complicated. 
Substituting for $I$, in Eq. (2la), the final expression for unsteady pressure field in the vicinity of the cascade is given by

$$
\begin{aligned}
& p_{c}^{\prime}(x, r, \theta, t)=\frac{N e^{i \pi / 4}}{2 \pi} \sum_{\mu=-\infty}^{\infty} \sum_{v=0}^{\infty} \sum_{t=0}^{\infty} \sum_{\ell=-\infty}^{\infty} A_{\mu \nu}
\end{aligned}
$$

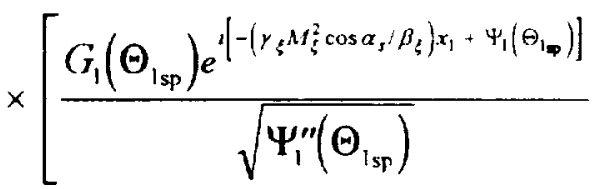

$$
\begin{aligned}
& \left.+\frac{G_{2}\left(\Theta_{2 \mathrm{sp}}\right) e^{\prime\left[\left(\gamma_{\xi} \Lambda \mu_{\xi}^{2} \cos \alpha_{s} / \beta_{\xi}\right) x_{2}+\Psi_{2}\left(\Theta_{2 \mathrm{~s}}\right)\right]}}{\sqrt{\Psi_{2}^{\prime \prime}\left(\Theta_{2 \mathrm{sp}}\right)}}\right] \\
& \times e^{i(\mu+2 \ell \pi / \varphi) \theta-\omega t]} \cos \left(l \pi \frac{r-\sigma}{1-\sigma}\right)
\end{aligned}
$$

The last step in obtaining the unsteady pressure distribution on the cascade involves evaluating $p_{c}^{\prime}$ on the airfoil surface.

Eq. (27) is the second principal result of this paper. It provides a closed form expression for computing the asymptotic behavior of the unsteady pressure distribution on an annular cascade in the high frequency limit. The next step in the analysis involves relating the cascade pressure distribution to the ducl acoustic modes.

The methodology of Green's function allows for the desired connection to be established between $p_{c}^{\prime}$ and the values of $C_{m}$ (in Eq. (13)). The mathematical statement of this connection is given by (see, for example, Meyer and Envia [1996])

$$
C_{m n}=\iint_{S} p_{c}^{\prime} \hat{e}_{s} \cdot \nabla\left(\Phi_{m n}(r) e^{i\left(k_{m n}^{ \pm} x+m \theta\right)}\right) d s
$$

where $\hat{\boldsymbol{e}}$ is the unit normal perpendicular to the surface of the reference airfoil. The integration is carried out over the surface of the reference airfoil. Once $C_{m n}$ 's are computed for each propagating mode according to Eq. (28), the duct acoustic field given by Eq. (13) is completely specified.

In a typical computation, a description of the incident gust field inside the annulus will be developed first. This will be done according to Eq. (A2.1) and then converted to an equivalent description in the local airfoil coordinate system as given by Eqs. (A2.2a through A2.2c). The resulting modal expansion will then be used as input to the cascade unsteady pressure formula (i.e., Eq. (27)). The result will be in turn integrated according to Eq. (28) to provide the modal pressure amplitudes $C_{m}$ 's. These will then be inserted in the expansion given by Eq. (13) to provide a description of the acoustic field inside the duct setup by the interaction of the incident gust with the annular cascade.

Eq. (13) gives a description of the acoustic field in terms of duct modal pressure. However, such quantities as acoustic power can be easily calculated using the applicable formulas from Eq. (13).

In principle, the steps outlined above will apply whether one is interested in tone noise or in broadband noise. But, in practice, some reformulation of the formulas will be required for broadband noise calculations. In particular, since the description of the turbulent gust can only be given in terms of spatial correlations, the formulas derived in this paper must be recast in terms of expected values of acoustic power instead of acoustic pressure. The methodology, however, will remain exactly the same. In an upcoming paper, detailed computations using the formulas derived here will be presented.

\section{CONCLUSIONS}

In summary, closed form asymptotic expressions for computing the noise field of an annular cascade inside a duct containing a uniform flow have been developed. By tailoring the analysis to the high frequency regime, numerical treatment of the equations involved has been avoided altogether.

There are two principal new results in this paper. The first is an alternative description of the duct acoustic modes that does not rely on the often-used Bessel function expansion. This results in simpler expressions for both the radial eigenvalues and eigenfinctions of the duct. In particular, the new representation provides an explicit approximate formul $\rightarrow$ for the radial eigenvalues obviating the need for sclutions of the transcendental annular duct eigenvatue equation. Also, the radial eigenfunctions are represented in terms of exponentials eliminating the numerical problems associated with generating the Bessel functions on a computer.

The second new element is the construction of an unsteady response model for an annular cascade 
using the single airfoil solution as the building block. The new construction allows for the boundary conditions on both the cascade and duct walls to be satisfied simultaneously adding a new level of realism to the noise calculations. Preliminary results were presented which demonstrate the utility and effectiveness of the new elements

Cascade discrete tone and broadband noise predictions using the new formulas will be presented in a future paper along with comparisons with results from simpler 2D-strip based models and rectilinear cascade models.

\section{APPENDIX 1}

\section{Asymptotic Solution of the Radial Eigenvalue Problem}

The WKB approximate solution of Eq. (8a) is given by

$\tilde{g}_{m}(r) \doteq \frac{1}{\sqrt{w(r)}}\left(C_{1} e^{i \int w(r) d r}+C_{2} e^{-i \int w(r) d r}\right)$

subject to

$$
\left.\left(\frac{d}{d r}-\frac{1}{2 r}\right) \widetilde{g}_{m}\right|_{r=\sigma, 1}=0
$$

where $C_{1}$ and $C_{2}$ are arbitrary constants and the symbol $\doteq$ denotes the approximate nature of the solution. The integration indicated in the phase of the exponential terms can be carried out explicitly. yielding

$$
\begin{gathered}
\psi(\kappa, r)=\int w(r) d r=\frac{1}{2} \sqrt{4(\kappa r)^{2}-4 m^{2}+1} \\
-\frac{1}{2} \sqrt{1-4 m^{2}}\left\{\log \left(\sqrt{1+\frac{4(\kappa r)^{2}}{1-4 m^{2}}}+1\right)\right. \\
\left.-\log \left(\sqrt{1-4 m^{2}} r\right)+2 \log (2)\right\}
\end{gathered}
$$

Assuming that $\kappa \gg>1$, Eq. (A1.2) can be simplified by expanding it at that limit. The simplified phase function to $O\left(x^{-2}\right)$ is given by

$$
\psi_{a}(\kappa, r) \cong \kappa r+\frac{4 m^{2}-1}{8 \kappa r}
$$

where the subscript $a$ indicates that Eq. (AI.3) is an approximation to Eq. (AI.2). The constant term in the expansion (i.e., the $O\left(x^{0}\right)$ term) is ignored since it only contributes a multiplicative constant to the expression for $\tilde{g}_{m}$ and therefore does not alter the subsequent results.

Substituting $\psi_{a}$ for $\psi$ in Eq. (Al.1a) and noting that $[w(r)]^{-1 / 2}=x^{-1 / 2}+O\left(x^{-2}\right)$, the boundary condition at $r=\sigma$ determines $C_{2}$ in terms of $C_{1}$ via

$$
\begin{aligned}
& C_{2}=C_{1} \frac{\varpi(\kappa, \sigma)}{\sigma^{*}(\kappa, \sigma)} e^{2 i \psi_{e}(\kappa, \sigma)} \\
& \omega(\kappa, \sigma)=2 \sigma \psi_{a}^{\prime}(\kappa, \sigma)+i
\end{aligned}
$$

where the prime denotes the derivative with respect to $r$ and the asterisk a complex conjugate quantity. Enforcing the boundary condition at $r=1$ and substituting for $C_{2}$ from Eqs. (A1.4a \& b) yields

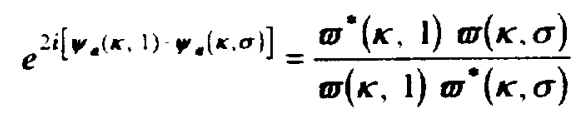

which is the equation that determines the nondimensional eigenvalue $\kappa$. In its present form, Eq. (A1.5) is transcendental in $\kappa$ and not amenable to an exact analytical solution. But if we take the logarithm of both sides and expand the resulting equation to $O\left(\kappa^{-2}\right)$. consistent with the level of approximation introduced in obtaining $\psi_{a}$, we find

$$
2\left[\psi_{a}(\kappa, 1)-\psi_{a}(\kappa, \sigma)\right]=\frac{(1-\sigma)}{\kappa}+2 n \pi
$$

where $n=0,1,2, \cdots$. In view of Eq. (Al.3), Eq. (A1.6) is quadratic in $x$ and can be readily solved to yield

$$
\kappa_{m n}=\frac{n \pi \sigma+\sqrt{(n \pi \sigma)^{2}+\sigma(1-\sigma)^{2}\left(2 m^{2}+3 / 2\right)}}{2 \sigma(1-\sigma)}
$$


where only the positive root has been retained since only the positive eigenvalues are relevant here. Nole that the pair of indices $m . n$ denotes a doubly infinite set. Eq. (AI.7) is a remarkably simple formula for the approximate eigenvalues of the annulus. The accuracy of this formula is investigated in the analysis section of this paper.

Inserting the expressions for $C_{2}$ and $\boldsymbol{K}_{m n}$ in Eq. (Al.la), we find the most general representation of $\tilde{g}_{m}(r)$ as

$$
\begin{aligned}
& \widetilde{g}_{m}(r) \doteq \sum_{n=0}^{\infty} \sqrt{\frac{1}{\kappa_{m n}}}\left(1-\frac{1-4 m^{2}}{16\left(\kappa_{m n} r\right)^{2}}\right) C_{m n}
\end{aligned}
$$

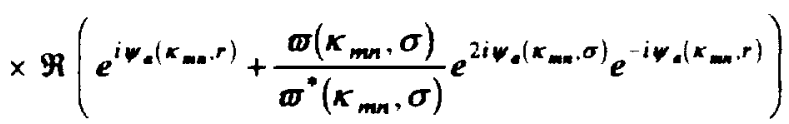

where $\Re$ denotes the real part of the quantity and $C_{m}$ is the constant $C_{1}$ renamed. To improve the accuracy of the eigenfunction approximation the next order term in the expansion of $[w(r)]^{-1 / 2}$ has also been kept resulting in the term in front of $C_{m m}$.

\section{APPENDIX 2}

\section{Asymptotic Solution of}

\section{The Single Airfoil Unsteady Pressure Distribution}

It is mathematically more convenient to solve this problem in terns of the acoustic velocity potential (denoted here by $\phi$ ) instead of the pressure. Once the solution is obtained, the pressure can be calculated from via the linearized momentum equation, i.e., $p^{\prime}=-\rho \frac{D_{0} \phi}{D t}$ where $\rho$ is the ambient density.

As was indicated earlier, the unsteady pressure response will be formulated and solved in the local coordinate system $(\xi, \eta, \zeta)$. It is, therefore. necessary to represent the incident gust in these coordinates. The most general representation. in the global coordinate system, of the relevant component of the convected gust is given by

$$
u_{g}(x, r, \theta, t)=\sum_{\mu=-\infty}^{\infty} \sum_{v=n}^{\infty} A_{\mu v} \Phi_{\mu v}(r) e^{i\left(k_{x} x+\mu \theta-\omega t\right)}
$$

where $\Phi_{\mu \nu}$ 's are the radial eigenfunctions of the duct (see Eq. (10a)). The axial wavenumber is given by $k_{x}=k / M$ owing to the requirement that the gust be convected by the mean flow (Recall that $k=\omega r_{T} / a_{0}$ ). $\boldsymbol{A}_{\mu \nu}$ 's denote the gust modal amplitudes.

Along the leading edge of the reference airfoil $\theta$ is zero. Setting $\theta=0$ in Eq. (A2.1), expressing the resulting equation in the local coordinate system, and expanding the $\zeta$ dependence in a Cosine Fourier series yields

$$
\begin{gathered}
u_{g}(\xi, \eta, \zeta, t)=\sum_{\mu=-\infty}^{\infty} \sum_{v=0}^{\infty} \sum_{t=0}^{\infty} a_{\mu \nu} e^{i\left(\gamma_{\xi} \xi+\gamma_{\eta} \eta-\theta t\right)} \\
\times \cos (l \pi \zeta) \\
\gamma_{\xi}=k_{x} \cos \alpha_{g}, \quad \gamma_{\eta}=-k_{x} \sin \alpha_{s} \\
a_{\mu \nu}=A_{\mu \nu} \int_{0}^{1} \Phi_{\mu \nu}[\sigma+(1-\sigma) \zeta] \cos (l \pi \zeta) d \zeta
\end{gathered}
$$

Eq. (A2.2a) provides the general modal representation of the incident gust in the local coordinate system $(\xi, \eta, \zeta)$.

In view of Eqs. (A2.2a) through (A2.2c), the mathernatical statement of the unsteady response of a single airfoil subject to a single incident gust mode is given by

$$
\begin{aligned}
& \left(\nabla^{2}-a_{0}^{-2} \frac{D_{0}^{2}}{D t^{2}}\right) \phi(\xi, \eta, \zeta, t)=0 \\
& \left.\frac{\partial \phi}{\partial \eta}\right|_{\eta=1}=-e^{i\left(\eta_{\xi} \xi-\boldsymbol{\omega} t\right)} \cos (l \pi \zeta) \text { on } 0 \leq \xi \leq c \\
& \left.p^{\prime}\right|_{\eta=0} ^{\eta=0}=0 \text { on } \xi \geq c
\end{aligned}
$$

where $c$ is the airfoil chord. The transverse wave number $\gamma_{\eta}$ does not enter these equations owing to the form of the boundary condition in Eq. (A2.3b). The convective derivative is given by $\frac{D_{0}}{D t}=\frac{\partial}{\partial t}+V_{\xi} \frac{\partial}{\partial \xi}$ where $V_{\xi}=U \cos \alpha_{s}$ is the speed of the uniform base 
flow, which now is aligned with the airfoil chord. The last equation enforces the continuity of the acoustic pressure downstream of the trailing edge. These equations are supplemented by the Sommerfeld radiation condition at infinity and the continuity of derivatives of off the airfoil.

The exact solution of Eq. (A2.3a), subject to all of the specified boundary conditions, can not be obtained in closed form. But, it has long been established that the solution may be developed as a convergent series $\phi=\phi_{1}+\phi_{2}+\cdots$ in which each term is a solution of Eq. (A2.3a) but with one of the two edges moved off to infinity. As a result, for each term in this sequence, some of the boundary conditions can be ignored leading to a simpler problem which can be solved in closed form. Landahl [1958] has shown that the series converges for all frequencies with the rate of convergence increasing rapidly as the frequency is increased. It will be shown later that, for our purposes, the first two terms in the sequence provide sufficient accuracy.

\section{The Leading Edge Solution}

The leading edge solution $\phi_{1}$ must satisfy Eq. (A2.3a) subject to

$$
\left.\frac{\partial \phi_{1}}{\partial \eta}\right|_{\eta-0}=-e^{i\left(\gamma_{\xi} \xi-\omega t\right)} \cos (l \pi \zeta) \text { on } 0 \leq \xi<\infty
$$

The introduction of the following change of variables

$$
\begin{aligned}
\phi_{1} & =\bar{\phi}_{1} e^{i\left[-\gamma_{\xi}\left(M_{\xi}^{2} / \beta_{\xi}\right) \xi^{\prime}-\omega t\right]} \cos (l \pi \zeta) \\
\xi^{\prime} & =\frac{\xi}{\beta_{\xi}} . \quad \beta_{\xi}=\sqrt{1-M_{\xi}^{2}}, \quad M_{\xi}=\frac{V_{\xi}}{a_{0}}
\end{aligned}
$$

simplifies Eqs. (A2.3a) and (A2.4) to

$$
\begin{aligned}
& \left(\frac{\partial^{2}}{\partial \xi^{\prime 2}}+\frac{\partial^{2}}{\partial \eta^{2}}+\chi^{2}\right) \bar{\phi}_{1}=0 \\
& \left.\frac{\partial \bar{\phi}_{1}}{\partial \eta}\right|_{\eta=0}=-e^{i\left(r_{\xi} / \beta_{\xi}\right) \xi^{\prime}} \text { on } 0 \leq \xi^{\prime}<\infty \\
& x=\sqrt{\frac{\gamma_{\xi}^{2} M_{\xi}^{2}}{\beta_{\xi}^{2}}-(l \pi)^{2}}
\end{aligned}
$$

A Fourier transform in $\xi^{\prime}$ reduces Eqs. (A2.6a) to an ODE whose general solution is given by

$$
\begin{aligned}
& \hat{\bar{\phi}}_{1}=\operatorname{sgn}(\eta) C_{1}(\lambda) e^{\sqrt{\lambda^{2}-x^{2}} \mid \eta n} \\
& \left.\frac{d \hat{\bar{\phi}}_{1}}{d \eta}\right|_{\eta=0}=-\frac{i}{\sqrt{2 \pi}\left(\lambda+\frac{\gamma_{\xi}}{\beta_{\xi}}\right)}
\end{aligned}
$$

where $\lambda$ is the Fourier transform variable, $\hat{\bar{\phi}}_{1}$ the transform of $\bar{\phi}_{1}$ and $\mathrm{Eq}$ ( $\mathrm{A} 2.7 \mathrm{~b}$ ) the transform of Eq. (A2.6b). The branch cuts of the square root in Eq. (A2.7a) are chosen so that the real part of the root is always positive. The unknown constant $C_{1}(\lambda)$ can be expressed in terms of known functions by substituting for $\hat{\bar{\phi}}_{1}$ in Eq. (A2.7b) and taking advantage of the requirement that the acoustic velocity be continuous everywhere. The resulting equation can then be easily solved through the use of the Wiener-Hopf technique (see Noble [1958]). The final expression is given by

$$
C_{1}(\lambda)=-\frac{1}{\sqrt{2 \pi} \sqrt{\frac{\gamma_{\xi}}{\beta_{\xi}}+\chi}\left(\lambda+\frac{\gamma_{\xi}}{\beta_{\xi}}\right) \sqrt{\lambda+\chi}}
$$

Substituting for $C_{1}(\lambda)$ in Eq. (A2.7a) and applying an inverse Fourier transform to the resulting expression yields the solution for $\bar{\phi}_{1}$. Reverting to the original dependent variable $\phi_{1}$ and computing the acoustic pressure (i.e., $p_{1}^{\prime}=-\rho \frac{D_{0} \phi_{1}}{D t}$ ) gives
$p_{1}^{\prime}\left(\xi^{\prime}, \eta, \zeta, t\right)=\frac{-i \rho V \operatorname{sgn}(\eta)}{2 \pi \beta_{\xi} \sqrt{\frac{\gamma_{\xi}}{\beta_{\xi}}+\chi}} \cos (l \pi \zeta)$

$\times e^{-i\left[\gamma_{\xi}\left(M_{\xi^{2}}{ }^{2} / \beta_{\xi}\right) \xi^{\prime}+\omega t\right]} \int_{-\infty}^{\infty} \frac{e^{-i \lambda \xi^{\prime}-\sqrt{\lambda^{2}-x^{2}|m|}}}{\sqrt{\lambda+\chi}} d \lambda$

The pole in Eq. (A2.8) does not appear in the expression for pressure since it represents a solenoidal (i.e., pressure-free) velocity. The integral in Eq. (A2.9) can be evaluated explicitly in terms of elementary functions. The development is straightforward and, therefore, only the final answer will be given here. The solution is 


$$
\begin{aligned}
& p_{1}^{\prime}\left(\xi^{\prime}, \eta, \zeta, t\right)=-\frac{\rho V \operatorname{sgn}(\eta) e^{i \pi / 4}}{\sqrt{\pi \beta_{\xi}} \sqrt{\gamma_{\xi}+\beta_{\xi} \chi} \sqrt[4]{\xi^{\prime 2}+\eta^{2}}} \cos \frac{v^{\prime}}{2} \\
& \times e^{j\left[x \sqrt{\xi^{\prime 2}+\eta^{2}-\left(r_{\xi} M_{\xi}^{2} / \beta_{\xi}\right) \xi^{\prime} \cdot \omega t}\right] \cos (l \pi \zeta)} \quad \text { (A2.10a) } \\
& v^{\prime}=\tan ^{\prime} \frac{\eta}{\xi^{\prime}}
\end{aligned}
$$

Eq. (A2.10a) gives the pressure field associated with the leading edge everywhere. Note that the pressure has a square root singularity at the leading edge and decays algebraically away from it. The airfoil chord. $c$, does not appear in the leading edge solution since the trailing edge was moved off to the downstream infinity. The pressure distribution on the airfoil (i.e., for $v^{\prime}=0$ ) is given by

$$
\begin{aligned}
& p_{1_{t}^{\prime}}^{\prime}\left(\xi^{\prime}, 0, \zeta, t\right) \\
& =\mp \frac{\left.\left.\rho V e^{i \pi / 4} e^{i\left\{\left[x-\left(r_{\xi} M_{\xi}^{2} / \beta_{\xi}\right)\right.\right.}\right) \xi^{\prime-\alpha \mu \ell}\right\}}{\sqrt{\pi \beta_{\xi}} \sqrt{r_{\xi}+\beta_{\xi} \chi} \sqrt{\xi^{\prime}}} \cos (l \pi \zeta)
\end{aligned}
$$

The plus sign indicates the pressure on the upper surface and the minus sign the pressure on the lower surface. Since the leading edge solution cannot satisfy the Kutta condition at the trailing edge (i.e., $p_{1}^{\prime}$ is not continuous there), the trailing-edge solution must be added to $p_{1}^{\prime}$ to rectify this shortcoming.

\section{The Trailing Edge Solution}

Now we let the leading edge move off to the upstream infinity and place the origin on the trailing edge. The new chordwise coordinate $\widetilde{\xi}$ is simply $\tilde{\xi}=\xi-c$. The trailing edge solution $\phi_{2}$ must also satisfy Eq. (A2.3a), but the appropriate boundary conditions are now given by

$$
\begin{aligned}
& \left.\frac{\partial \phi_{2}}{\partial \eta}\right|_{\eta=11}=0 \text { on } \tilde{\xi} \leq 0 \\
& \left.\left(p_{1}^{\prime}-\rho \frac{D_{0} \phi_{2}}{D \eta}\right)\right|_{\eta-0} ^{\eta-0^{+}}=0 \text { on } \tilde{\xi} \geq 0
\end{aligned}
$$

where $\boldsymbol{p}_{1}^{\prime}$ in Eq. (A2.12b) is the leading edge solution (i.e.. Eq. (A2.11)) expressed in the shifted chordwise coordinate $\tilde{\xi}$.
Following the same procedure that was outlined for the leading edge solution, first a reduced potential is introduced,

$$
\phi_{2}=\bar{\phi}_{2} e^{\left.i \mid-r_{\xi}\left(M_{\xi}^{2} / \beta_{\xi}\right) \dot{\xi}^{\prime}-\boldsymbol{\omega t}\right]} \cos (l \pi \zeta)
$$

and then a Fourier transform in $\tilde{\xi}^{\prime}$ (i.e.. $\tilde{\xi}^{\prime}=\tilde{\xi} / \beta_{\xi}$ ) is applied allowing the formal solution for $\hat{\bar{\phi}}_{2}$ (the Fourier transform of $\bar{\phi}_{2}$ ) to be found. The result is

$$
\hat{\bar{\phi}}_{2}=\operatorname{sgn}(\eta) C_{2}(\lambda) e^{\sqrt{\lambda^{2}-x^{2}}|\eta|}
$$

subject to the transformed version of Eq. (A2.12b);

$$
\begin{aligned}
& \frac{\left.\sqrt{2} i e^{i\left\{\left[x-\left(r_{\xi} M_{\xi}^{2} / \beta_{\xi}\right)\right] c^{\prime}+\pi / 4\right.}\right\}}{\pi \sqrt{\frac{\gamma_{\xi}}{\beta_{\xi}}+\chi}} \int_{0}^{\infty} \frac{e^{i(\lambda+x) \xi^{\prime}}}{\sqrt{\xi^{\prime}+c^{\prime}}} d \tilde{\xi}^{\prime} \\
& +\left.\left(\lambda+\frac{\gamma_{\xi}}{\beta_{\xi}}\right) \hat{\bar{\phi}}_{2}\right|_{\eta=0^{-}} ^{\eta=0^{+}}=0 \text { on } \tilde{\xi} \geq 0
\end{aligned}
$$

where the terms on the left-hand side are the Fourier transforns of the acoustic pressures associated with $\phi_{1}$ and $\phi_{2}$. respectively, and $c^{\prime}=c / \beta_{\xi}$.

The integral in Eq. (A2.14b) can be evaluated explicitly in terms of the Fresnel Integrals. But, since we are only interested in the pressure distribution upstream of the trailing edge, it suffices to determine the behavior of the integral near the trailing edge (i.e.. for $\tilde{\xi}^{\prime}=0$ ). Expanding the denominator of the integra id and integrating term by term yiclds the power series

$$
\begin{array}{r}
\int_{0}^{\infty} \frac{e^{i\left(\lambda+x \mid \xi^{\prime}\right.}}{\sqrt{\xi^{\prime}+c^{\prime}}} d \tilde{\xi}^{\prime}=\frac{i}{\sqrt{c^{\prime}}(\lambda+\chi)}+\frac{1}{2 \sqrt{c^{\prime 3}}(\lambda+\chi)^{2}} \\
+O\left((\lambda+\chi)^{3}\right)
\end{array}
$$

of whch only the leading term is kept in the subsequent analysis. This approximation simplifies the algebra significantly while still guaranteeing that the Kutta condition is satisfied at the trailing edge. 
In a slightly more complicated Wiener-Hopf analysis, the unknown constant $C_{2}(\lambda)$ in Eq. (A2.14a) is found to be

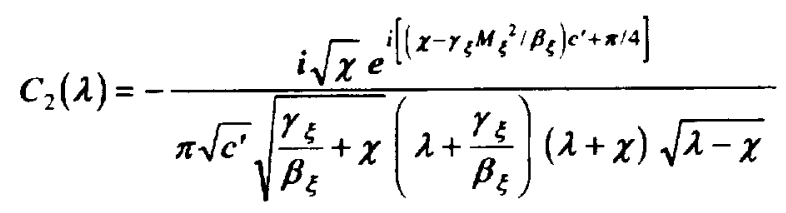

With $C_{2}(\lambda)$ known. Eq. (A2.14a) can be formally inverted. Switching back to the original dependent variable $\phi_{2}$ and computing the acoustic pressure we find

$$
\begin{aligned}
& p_{2}^{\prime}\left(\widetilde{\xi}^{\prime}, \eta, \zeta, t\right)=\frac{\rho V \operatorname{sgn}(\eta) \sqrt{\chi} e^{\left.i x \cdot \gamma_{\xi}\left(M_{\xi}^{2} / \beta_{\xi}\right)\right] c^{\prime}+i \pi^{\prime 4}}}{\beta_{\xi} \sqrt{2 \pi^{3} c^{\prime}} \sqrt{\gamma_{\xi}+\chi}} \\
& \times e^{i\left[-\gamma_{\xi}\left(M_{\xi}^{2} / \beta_{\xi}\right) \tilde{\xi}^{\prime}-\omega t\right]} \cos (l \pi \zeta) \int_{-\infty}^{\infty} \frac{e^{-i \lambda \tilde{\xi}^{\prime}-\sqrt{\lambda^{2}-\chi^{2}}|\eta|}}{\sqrt{\lambda-\chi}(\lambda+\chi)} d \lambda
\end{aligned}
$$

The above integral can be evaluated explicitly. After a fair amount of algebra. the final result, expressed in the leading edge coordinates $\left(\xi^{\prime}, \eta, \zeta\right)$, is given by

$$
\begin{aligned}
& p_{2}^{\prime}\left(\xi^{\prime}, \eta, \zeta . t\right) \\
& =\frac{\rho V \operatorname{sgn}(\eta) e^{i x / 4}}{\sqrt{\pi \beta_{\xi} c^{\prime}} \sqrt{\gamma_{\xi}+\beta_{\xi} \chi}} e^{i\left\{\left[x-\left(r_{\xi^{M}}{ }_{\xi^{2} / \beta_{\xi}}\right)\right] \xi^{\prime}-\omega r^{\prime}\right\}} \cos (l \pi \zeta) \\
& \times\left[\varepsilon_{\xi^{\prime}}+\frac{2 e^{-i \pi / 4}}{\sqrt{\pi}} F\left(\sqrt{x\left(\sqrt{\left(c^{\prime}-\xi^{\prime}\right)^{2}+\eta^{2}}+c^{\prime}-\xi^{\prime}\right)}\right)\right] \\
& \text { (A2.18a) } \\
& \varepsilon_{\xi^{\prime}}=\left\{\begin{array}{lll}
0 & \text { if } & \xi^{\prime}-c^{\prime} \leq 0 \\
1 & \text { if } & \xi^{\prime}-c^{\prime}>0
\end{array}\right.
\end{aligned}
$$

where $F(z)=\int_{z}^{\infty} e^{i \alpha^{2}} d \alpha$ is the Fresnel Integral. Note that, unlike the leading edge solution, the trailing edge solution is not singular.

Evaluating $p_{2}^{\prime}$ on the airfoil (i.e., $\boldsymbol{\eta}=0$ ) and combining it with Eq. (A2.11), yields the two-term representation of the unsteady pressure distribution on the airfoil. The result is

$$
\begin{aligned}
& \boldsymbol{p}_{ \pm}^{\prime}\left(\xi^{\prime}, 0, \zeta, t\right)= \\
& \mp \frac{\rho V e^{i \pi / 4}}{\sqrt{\pi \beta_{\xi}} \sqrt{\gamma_{\xi}+\beta_{\xi} \chi}} e^{i\left\{\left[\boldsymbol{x}\left(\gamma_{\left.\left.\xi^{M} \xi_{\xi}^{2} / \beta_{\xi}\right)\right] \xi^{\prime}-\Delta x}\right\}\right.\right.} \cos (l \pi \zeta) \\
& \quad \times\left\{\frac{1}{\sqrt{\xi^{\prime}}}-\frac{2 e^{-i \pi / 4}}{\sqrt{\pi c^{\prime}}} F\left(\sqrt{2 \chi\left(c^{\prime}-\xi^{\prime}\right)}\right)\right\}
\end{aligned}
$$

The combined solution satisfies the Kutta condition at the trailing edge (i.c., at $\xi^{\prime}=c^{\prime}$ ) since $F(0)=\sqrt{\pi} e^{i \pi / 4} / 2$.

In Figure 4 magnitude of the unsteady surface pressure as predicted by Eq. (A2.19) is plotted for typical (high) reduced frequency and Mach number values. The corresponding plots for $\boldsymbol{p}_{1_{t}}^{\prime}\left(\xi^{\prime}, 0, \zeta, t\right)$ and $p_{2,}^{\prime}\left(\xi^{\prime}, 0, \zeta, t\right)$ are also shown for comparison. For this calculation the gust is assumed to be uniform along the span. Therefore, Figure $\&$ effectively shows the chordwise pressure distribution.

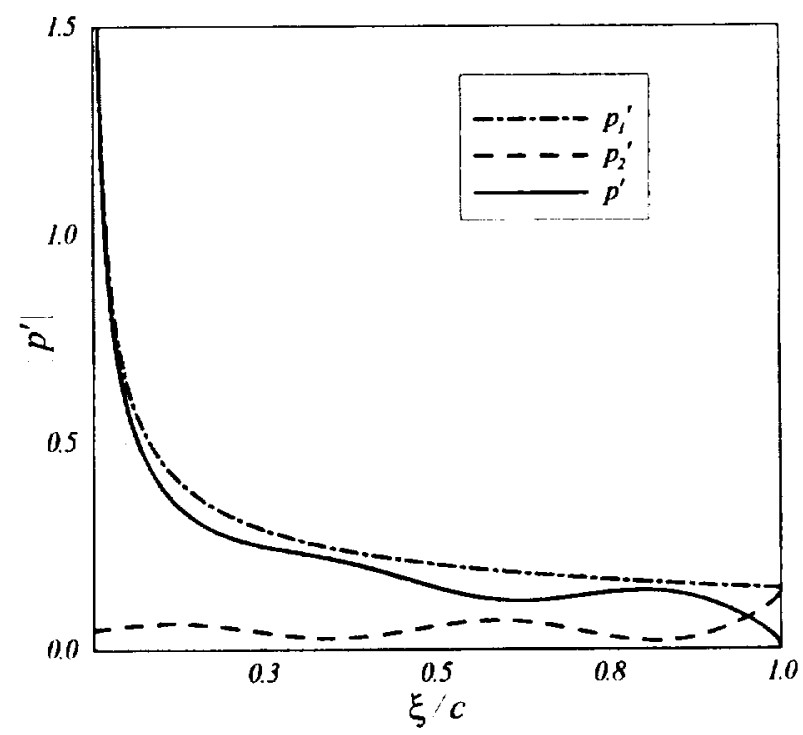

Figure 4. Typical chordwise unsteady pressure distribution on a single airfoil. $\boldsymbol{p}_{1}^{\prime}$ and $\boldsymbol{p}_{2}^{\prime}$ are leading edge and trailing edge solutions, and $\boldsymbol{p}^{\prime}$ the combined solution. $\left(\gamma_{\xi}, \gamma_{\zeta}\right)=(10,0)$ and $M_{\xi}=0.5$ for this calculation. 

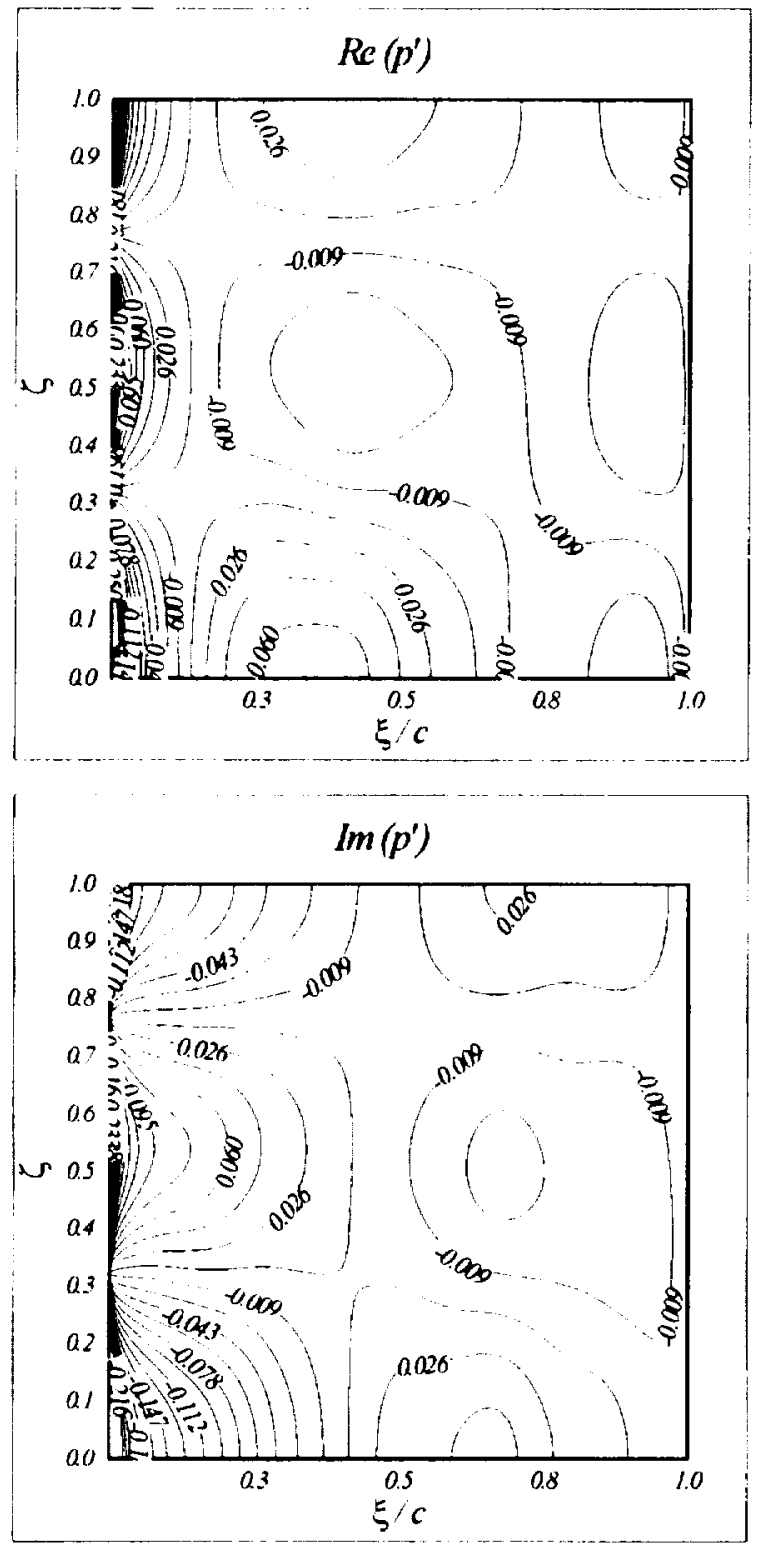

Figure 5. P'redicted real and imaginary parts of the 3D unsteady pressure distribution on a single airfoil for a gust with radial structure. The gust has the form $u_{g}=\Phi_{02}(r) e^{i \gamma \xi}$, with $\gamma_{\xi}=22$ and $M_{\xi}=0.5$

Note that $\boldsymbol{p}_{2}^{\prime}$ 's primary contribution is in the vicinity of the trailing edge where it enforces the Kutta condition by canceling out the leading edge contribution there. Everywhere else it is only a sinall correction to the leading edge solution. The third term in Landahl's series will be correspondingly smaller in comparison with the leading edge solution. At high frequencies. therefore, the additional complexity incurred by adding the third term is hardly justified for a very small improvement in the accuracy of the

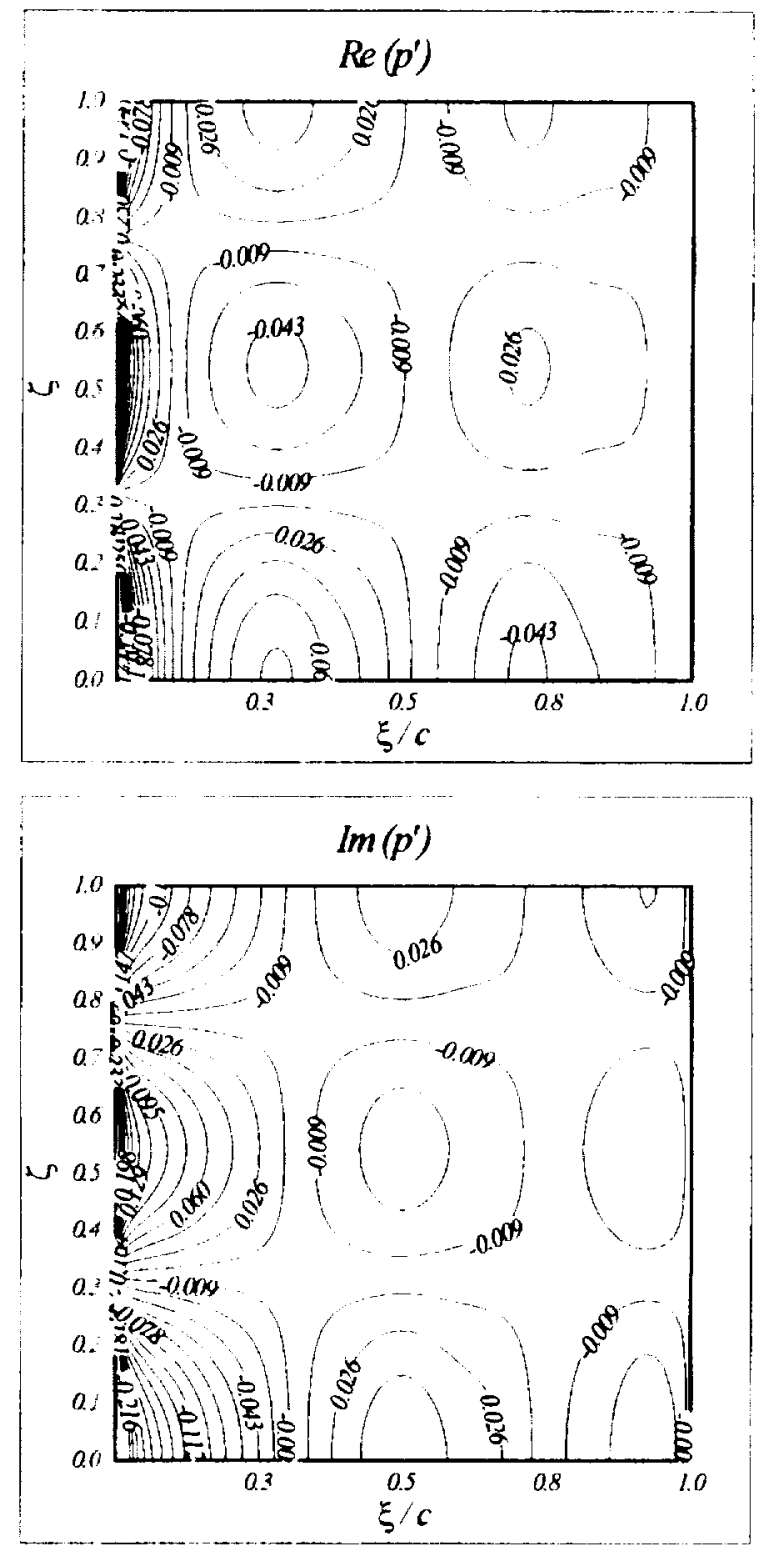

Figure 6. Predicted real and imaginary parts of the unsteady pressure distribution for a single airfoil using 21)strip approximation. The gust parameters are the same as those in Figure 5

solution. Hence, we shall truncate the series at this point and use the two-term representation in the subsequent analysis.

In Figure 5 the predicted real and imaginary parts of the unsteady pressure distribution for a single airfoil as given by Eq. (A2.19)) are shown for a more realisti: gust distribution given by $u_{g}=\Phi_{62}(r) e^{i \gamma_{\xi} \xi}$ with $\gamma_{\xi}=22, \sigma=0.5$ and $M_{\xi}=0.5$. The radial structure of the gust means that there will be a 
spectrum of spanwise wavenumbers as dictated by Eq. (A2.2a) and their associated spanwise amplitudes as given by $\mathrm{Eq}$. (A2.2c). Only the first five terms in that series were taken into account for this calculation since the higher order terms result in cut-off waves (i.e., $x$. given by $\mathrm{Eq}$. (A2.6c), is complex for $l \geq 5$ ). The corresponding amplitudes of the spanwise modes are given by $(0.067,0.127,0.375,-0.028,-0.004)$. For the sake of comparison the corresponding 2D-strip approximation to the unsteady pressure distribution is plotted in Figure 6. For this calculation, the variation of the gust amplitude along the span was taken into account but not its spanwise wavenumber spectrum. While overall structure of the $3 \mathrm{D}$ and 2D-strip distributions look similar, they exhibit differences in their detail. A discussion of the importance of these differences and their effect on the noise fields is postponed to a later paper

In order to utilize the formulas developed in this appendix in the analysis outlined in the report, it is necessary to express them in the duct coordinates. After some simplification and grouping of terms, the final expressions are given by

$$
\begin{array}{r}
p_{0 j}^{\prime}(x, r, \theta, t)=\sum_{\mu=-\infty}^{\infty} \sum_{r=0}^{\infty} \sum_{t=0}^{\infty} A_{\mu u} G_{j}(x, r, \theta) \cos \left(I \pi \frac{r-\sigma}{1-\sigma}\right) \\
\times e^{i\left[r_{j}(x, r, \theta)-\omega t\right]} \quad(\mathrm{A} 2,20 \mathrm{a})
\end{array}
$$

$$
\begin{aligned}
\tau_{j}(x, r, \theta)= & \chi R_{j}(x, r, \theta) \\
& -\gamma_{\xi} \frac{M_{\xi}^{2}}{\beta_{\xi}}\left(x \cos \alpha_{s}+r \theta \sin \alpha_{s}\right)
\end{aligned}
$$

$$
A_{\mu \downarrow}=\frac{a_{\mu \kappa} \rho V \operatorname{sgn}\left[\sin \left(g-\alpha_{s}\right)\right] e^{i \pi / 4}}{\sqrt{\pi \beta_{\xi}} \sqrt{\gamma_{\xi}+\beta_{\xi} \chi}}
$$

$G_{1}(x, r, \theta)=-\frac{\cos (g / 2)}{\sqrt{R_{1}}}$

$G_{2}(x, r, \theta)=\frac{2 e^{i\left[x\left(-R_{2}+x \cos \alpha_{s}+r \theta \sin \alpha_{s}\right)-y_{\xi} \frac{M_{\xi}^{2}}{\beta_{\xi}^{2} c-\pi / 4}\right]}}{\sqrt{\pi c^{\prime}}}$

$\times F\left[\chi\left(R_{2}+(x-c) \cos \alpha_{s}+r \theta \sin \alpha_{s}\right) / \beta_{\xi}\right]$

$R_{j}=\sqrt{x_{j}^{2}+(r \theta)^{2}}, \quad x_{j}=x-(j-1) c$

$$
\vartheta=\tan ^{-1}\left(\frac{-x \sin \alpha_{s}+r \theta \cos \alpha_{s}}{x \cos \alpha_{s}+r \theta \sin \alpha_{s}}\right)
$$

where $j=1$ gives the leading edge solution and $j=2$ the trailing edge solution. Eq. (A2.20a) represent the unsteady pressure field in the vicinity of a single airfoil bound by the walls of the duct with the index " 0 " on $\boldsymbol{p}_{0}^{\prime}$, indicating that the solution applies to the zeroth airfoil.

\section{REFERENCES}

1. Carrier, G.F., Krook, M. and Pcarson, C.E., Functions of a Complex Variable, McGraw-Hill, Inc. New York, 1966.

2. Envia, E. Huff, D., and Morrison, C.R. "Analytical Assessment of Stator Sweep and Lean in Reducing Rotor-Stator Tone Noise," AIAA Paper 96-1791, May 1996.

3. Envia. E. and Kerschen, E.J., "Noise Generated by Convected Gusts Interacting with Swept Airfoil Cascades." AIAA Paper No. 84-1872, July 1986

4. Glegg, S.A.L., "Airfoil Self Noise Generated in a Cascade." AlAA Paper 96-1739, May 1996.

5. Goldstein, M.E., Aeroacosutics, McGraw-Hill. Inc., New York, 1976, pp. 222-248.

6. Kobayashi, H., "Three-Dimensional Effects on Pure Tone Fan Noisc Due to Inflow Distortion," AIAA Paper 78-1120. July 1978.

7. Landahl, M.T.. "Theoretical Studies of Unsteady Transonic Flow - IV. The Oscillating Rectangular Wing with Control Surfaces," Aeronautical Research Institute of Sweden (FFA), Report No 80, 1958)

8. Mani, R, "Isolated Rotor Noise due to Inlet Distortion or Turbulence." NASA-CR 2479 , October 1973.

9. Meyer, H.D. and Envia E., "Aeroacoustic Analysis of Turbofan Noise Generation," NASACR 4715, March 1996

10. Namba, M., "Three-Dimensional Analysis of Blade Force and Sound Generation for An Annular Cascade in Distorted Flows." $J$. Sound \& libration, vol. 50. No. 4, Feb. 22, 1977. 
11. Nobel, B.. Methods Based on the Wiener-Hopf Technique, Pergamon Press, New York, 1958

12. Olver, F. W.J., Asymptotics and Special Functions, Academic Press, New York, 1974.

13. Schulten, J.B.H.M., "Sound Generation by Ducted Fans and Propellers as a Lifting Surface Problem," Ph.D. Thesis. 1993.

14. Tyler. L.M. and Sofrin, T.G., "Axial Flow Compressor Noise Studies," Trans. SAE. No. 70. pp. 309-332.1962.

15. Ventres. C.S.. Theobald, M.A., Mark. W.D., "Turbofan Noise Generation, Volume I: Analysis." NASA-CR 167951, July 1982. 


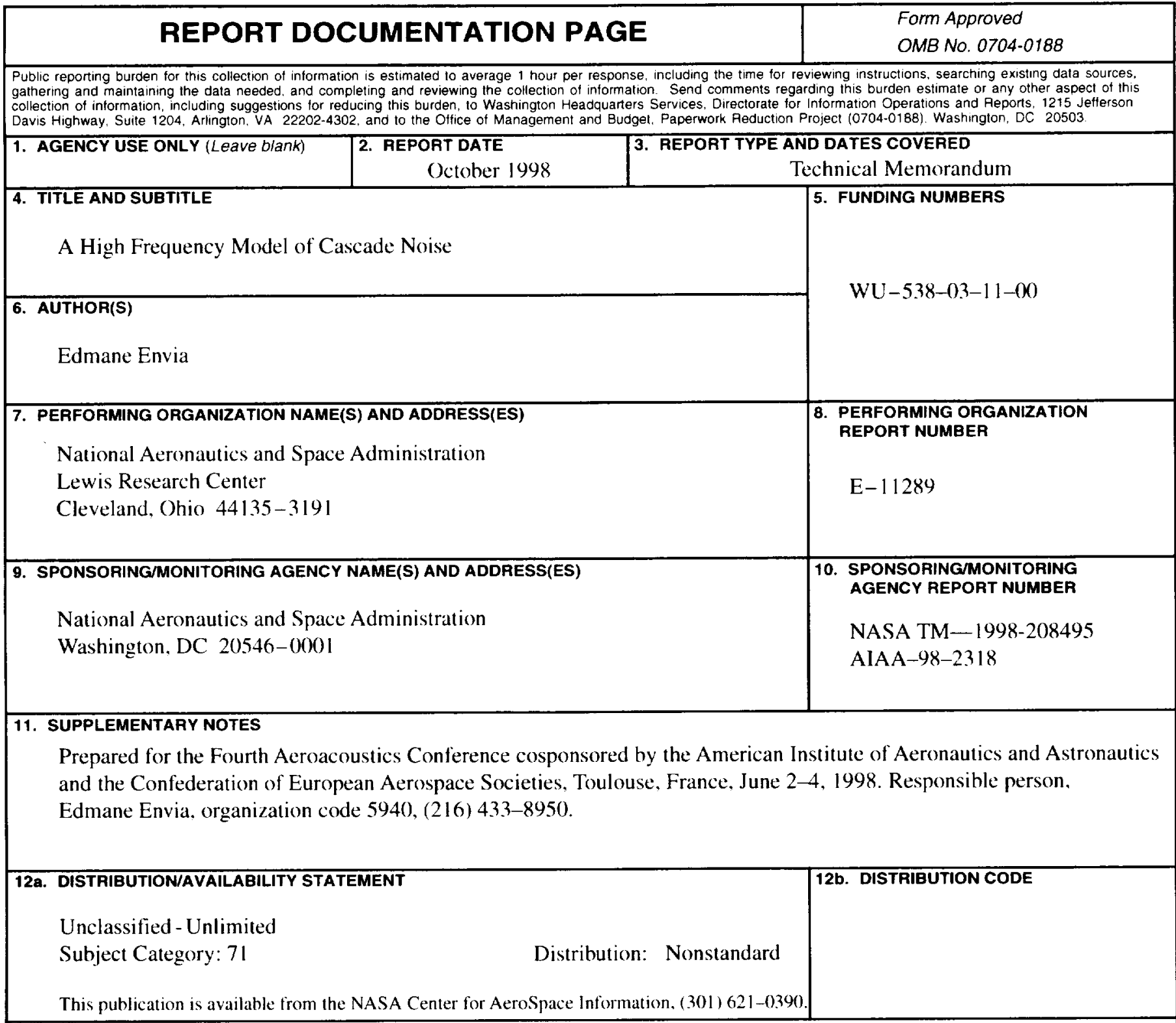

13. ABSTRACT (Maximum 200 words)

Closed form asymptotic expressions for computing high frequency noise generated by an annular cascade in an infinite duct containing a uniform flow are presented. There are two new elements in this work. First, the annular duct mode representation does not rely on the often-used Bessel function expansion resulting in simpler expressions for both the radial eigenvalues and eigenfunctions of the duct. In particular, the new representation provides an explicit approximate formula for the radial eigenvalues obviating the need for solutions of the transcendental annular duct eigenvalue equation. Also, the radial eigenfunctions are represented in terms of exponentials eliminating the numerical problems associated with generating the Bessel functions on a computer. The second new element is the construction of an unsteady response model for an annular cascade. The new construction satisfies the boundary conditions on both the cascade and duct walls simultaneously adding a new level of realism to the noise calculations. Preliminary results which demonstrate the effectiveness of the new elements are presented. A discussion of the utility of the asymptotic formulas for calculating cascade discrete tone as well as broadband noise is also included.

14. SUBJECT TERMS

Cascade noise; Noise prediction; Asymptotic methods: High-frequency; Broadband noise

\begin{tabular}{|c|c|}
\hline $\begin{array}{c}\text { 17. SECURITY CLASSIFICATION } \\
\text { OF REPORT } \\
\text { Unclassified }\end{array}$ & $\begin{array}{c}\text { 18. SECURITY CLASSIFICATION } \\
\text { OF THIS PAGE } \\
\text { Unclassified }\end{array}$ \\
\hline
\end{tabular}

19. SECURITY CLASSIFICATION OF ABSTRACT

NSN 7540-01-280-5500

\begin{tabular}{|l|}
\hline $\begin{array}{c}\text { 15. NUMBER OF PAGES } \\
22\end{array}$ \\
16. PRICE CODE \\
A0 3 \\
\hline 20. LIMITATION OF ABSTRACT
\end{tabular}


